\title{
INFLUENCIA DE LOS TIPOS DE LACTANCIA MATERNA EN LA FUSION DE LOS PROCESOS PALATINOS EN NEONATOS CON FISURA LABIOPALATINA
}

\author{
Yoshio Dakusaku López¹, Américo Munayco Magallanes²
}

\begin{abstract}
RESUMEN
Objetivo. Determinar la relación entre la lactancia materna exclusiva y fusión de los procesos palatinos, en neonatos con fisura labiopalatina unilateral. Materiales y métodos. Estudio longitudinal y de cohorte, conformado por 2 grupos: A) grupo de estudio: 4 neonatos con fisura labiopalatina unilateral, que recibieron lactancia materna exclusiva. B) grupo control: 4 neonatos con fisura labiopalatina unilateral, que recibieron lactancia materna no exclusiva. Para determinar el nivel de fusión de los procesos palatinos, se registró el maxilar superior con una impresión en 3 periodos (inicio, día 10 y día 20). En cada modelo obtenido se midió con un calibrador digital la distancia existente entre ambos procesos palatinos, siendo el resultado obtenido representativo del nivel de fusión de los procesos palatinos. Resultados. El nivel de fusión de los procesos palatinos en los neonatos que recibieron LME, desde el inicio del seguimiento al día 20 logró un aumento total de $2,44 \mathrm{~mm}$; mientras que en el grupo que recibió LMNE logró un aumento total de 2,28 $\mathrm{mm}$. Sin embargo, en ambos grupos la diferencia de promedios no fue estadísticamente significativa. Conclusión. La LME es la que estimula una mayor fusión de los procesos palatinos en comparación a la LMNE; sin embargo con diferencia estadísticamente no significativa.
\end{abstract}

Palabras clave: Lactancia materna exclusiva y no exclusiva; Fisura labiopalatina; Fusión de procesos palatinos.

\section{INFLUENCE OF THE TYPES OF BREASTFEEDING IN THE FUSION OF THE PALATAL PROCESSES IN INFANTS WITH CLEFT LIP AND PALATE}

\begin{abstract}
Objective. Determine the relationship between exclusive breastfeeding and fusion of palatal processes, in neonates with unilateral cleft lip palate. Materials and methods. Longitudinal and cohort study, conformed of 2 groups: A) group of study: 4 neonates with unilateral cleft lip palate with exclusive breastfeeding; B) control group: 4 neonates with unilateral cleft lip palate with non-exclusive breastfeeding. To determine the level of fusion of the palatal processes was registry the maxilla with an impression in 3 periods (start, day 10 and day 20). In each model obtained, was measure with a digital caliper the distance between the palatal processes. Results. The level of fusion of the palatal processes in the neonates with exclusive breastfeeding, from start to the day 20 increased $2,44 \mathrm{~mm}$. The level of fusion of palatal processes, in the neonates with non-exclusive breastfeeding, from start to the day 20 increased $2,28 \mathrm{~mm}$. Although with difference statistically doesn't significative. Conclusion. The exclusive breastfeeding stimulates higher fusion of the palatal processes, in compared the non-exclusive breastfeeding, although the difference is not statistically significative.
\end{abstract}

Key words: Exclusive and non-exclusive breastfeeding; Cleft lip palate; Fusion of palatal crests.

\section{INTRODUCCIÓN}

La fisura labiopalatina es la malformación congénita más frecuente que se produce durante el desarrollo facial embrionario. El labio leporino es una alteración embriológica que habitualmente afecta al labio superior, caracterizado por la ausencia de fusión de las dos partes del labio en una sola estructura ${ }^{1,2}$; mientras que la fisura palatina es una alteración embriológica caracterizada por la ausencia de fusión completa de los procesos palatinos ${ }^{2,3}$; la cual es definida como la aproximación, mesodermización y consolidación remodeladora que se genera entre ambos procesos palatinos y entre estas con el paladar primario y el vómer ${ }^{4,5}$.

Si consideramos que la leche materna es el único factor importante en la lactancia materna, entonces daría lo mismo que el lactante lacte directamente de la madre

Cirujano Dentista. Egresado de la Facultad de Odontología de la Universidad San Martín de Porres.

Magister, Cirujano Dentista. Especialista, Docente de la Facultad de Odontología de la Universidad San Martín de Porres. Docente Ordinario de la Facultad de Odontología de la Universidad Nacional Federico Villarreal. Odontólogo Asistencial del Instituto Nacional Materno Perinatal ( Ex Maternidad de Lima). 
o que se alimente por medio de un biberón, con tal que su contenido sea leche materna; pero si consideramos que además de la leche materna, también, la correcta actividad muscular y todo el proceso neurobiológico que se produce únicamente cuando el lactante se alimenta directamente de la madre, es indispensable para estimular su desarrollo, podemos entender la importancia de la lactancia materna exclusiva (LME), que es cuando el lactante se alimenta exclusivamente de leche materna directamente de la madre, sin complementos de ningún tipo (fórmula) que son administrados por medio de un biberón ${ }^{6,7}$. La lactancia materna no exclusiva (LMNE) es aquella en la que el lactante jamás a lactado directamente de la madre y se alimenta con leche materna o con fórmula o ambas pero administradas por medio de un biberón. También es cuando el lactante se alimenta directamente de la madre pero además se alimenta con leche materna o con fórmula o ambas, pero administradas por medio de un biberón.

Donato y Cols, mencionan que durante la LME el lactante utiliza por lo menos 60 veces mas energía ingiriendo su alimento en comparación a aquellos que se alimentan mediante biberón; siendo el esfuerzo y toda la actividad que se produce en el sistema estomatognático durante la $\mathrm{LME}$, indispensable para estimular un correcto desarrollo óseo, muscular y articular. Martin y Mohor, mencionan que la actividad muscular que se realiza durante la LME es tan indispensable para el crecimiento como la alimentación en sí. Blanco y Cols, encontraron asociación significativa entre el paladar profundo con un periodo de LME menor a 6 meses, eso se debe a que cuando el lactante se alimenta mediante un biberón, el chupo al ser alargado, grueso y duro, aprisiona a la lengua en el piso de la boca y durante el reflejo de deglución la lengua la proyecta hacia adelante; mientras que cuando el lactante se alimenta con LME, durante el reflejo de deglución la lengua la proyecta hacia arriba, generando una presión benéfica sobre el paladar la cual actúa como estimulo para el desarrollo del maxilar superior ${ }^{8}$. Garcez L. W, menciona que en presencia de fisura labiopalatina la lactancia materna exclusiva estimula un mejor desarrollo estructural y funcional del sistema estomatognático, además la leche materna mejora el sistema inmunológico y disminuye la tendencia a infecciones gracias a su contenido de inmunoglobulinas, lactoferrina, lisozima y factor bifidus ${ }^{9}$.

El presente estudio pretende determinar si la lactancia materna exclusiva estimula y favorece la fusión de los procesos palatinos, en neonatos que nacen con fisura labiopalatina, basándose en las múltiples ventajas de la lactancia materna exclusiva.

\section{MATERIALES Y MÉTODOS}

Tipo de investigación: Es un estudio correlacional ya que evaluó los efectos que genera la lactancia materna exclusiva (en el primer grupo) y no exclusiva (en el segundo grupo) en los procesos palatinos a nivel de la hendidura.

Diseño de investigación: El presente estudio es longitudinal y de cohorte ya que determina y compara en diferentes periodos de tiempo el nivel de fusión de los procesos palatinos, en neonatos con fisura labiopalatina unilateral sometidos a lactancia materna exclusiva y no exclusiva. Es un estudio prospectivo ya que parte de la causa que son aquellos neonatos con fisura labiopalatina unilateral sometidos a lactancia materna exclusiva y no exclusiva, para observar los efectos que se generan en los procesos palatinos a nivel de la hendidura. Es un estudio no experimental ya que evaluó los efectos que genera la lactancia materna exclusiva y no exclusiva (variable independiente) en la fusion de los procesos palatinos (variable dependiente) tal y como se da naturalmente, es decir, sin ninguna manipulación de la variable independiente.

Población y muestra: La población está constituida por 9 neonatos con fisura labiopalatina unilateral nacidos en el Institutos Nacional Materno Perinatal (INMP) entre Julio del 2010 a Febrero del 2011. La muestra esta constituida por 8 neonatos con fisura labiopalatina unilateral. El presente estudio al ser clínico y al requerir de libertad para escoger la muestra según criterio, se trabajó con un muestreo no probabilístico por conveniencia.

Definición de grupos: Comprendió los siguientes grupos: A) grupo de estudio: 4 neonato con la presencia de la patología a investigar según código CIE 10: Q 37,5 que recibieron lactancia materna exclusiva. B) grupo control: 4 neonato con la presencia de la patología a investigar según código $\mathrm{CIE}$ 10: Q 37,5 que recibieron lactancia materna no exclusiva.

Criterios de inclusión: se aceptaron neonatos que presentaron fisura labiopalatina unilateral pudiendo ser del lado derecho o izquierdo, de ambos géneros, no mayores de 8 días de edad y que se encontraron clínicamente sanos.

Criterios de exclusión: se rechazaron a aquellos neonatos que presentaron fisura labiopalatina bilateral, neonatos con fisura labial aislada o fisura palatina aislada, a aquellos neonatos cuya fisura palatina no comprometió tejido óseo (solo mucosa). Se rechazaron a neonatos mayores de 8 días de edad, a neonatos que presentaron alguna otra malformación o algún compromiso sistémico que pudo interferir con los resultados (fisura labiopalatina sindromática).

\section{Técnica}

Los neonatos nacidos con fisura labiopalatina unilateral (figura 1) que fueron trasferidos por interconsulta al consultorio de estomatología del INMP y que cumplieron los requisitos mencionados en los criterios de inclusión, fueron seleccionados para la presente investigación. Los padres firmaron un consentimiento informado en el 


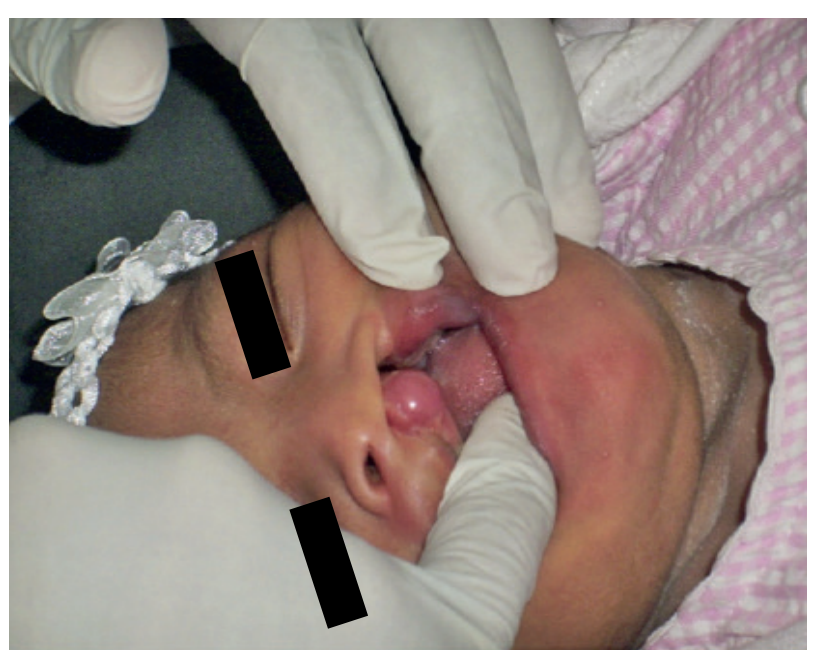

Figura 1. Neonato con fisura labiopalatina unilateral.

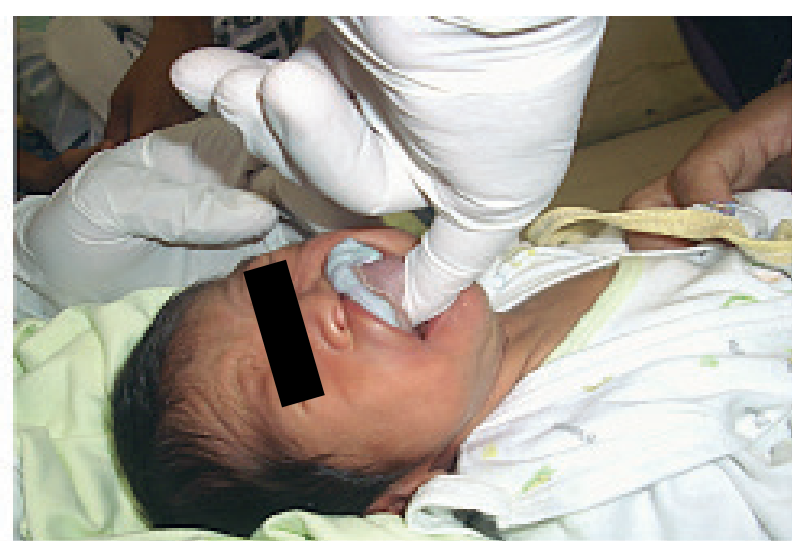

Figura 3. Registro del maxilar superior.

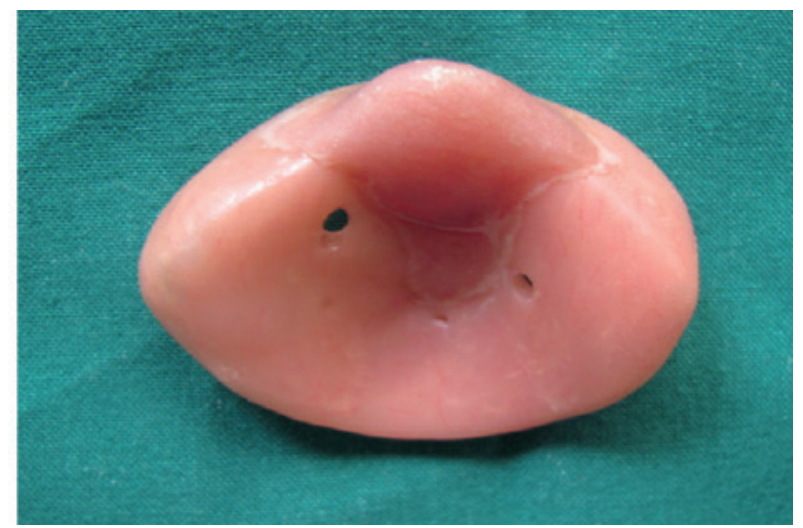

Figura 5. Cubeta para impresión.

cual se les explicó los procedimientos y controles que se realizarían, se explicó las ventajas que brinda controlar el desarrollo y crecimiento del neonato portador de fisura labiopalatina unilateral.

Para determinar el nivel de fusión de los procesos palatinos, se registró el maxilar superior (Figuras 2, 3 y 4) mediante una impresión con silicona pesada Zetaplus

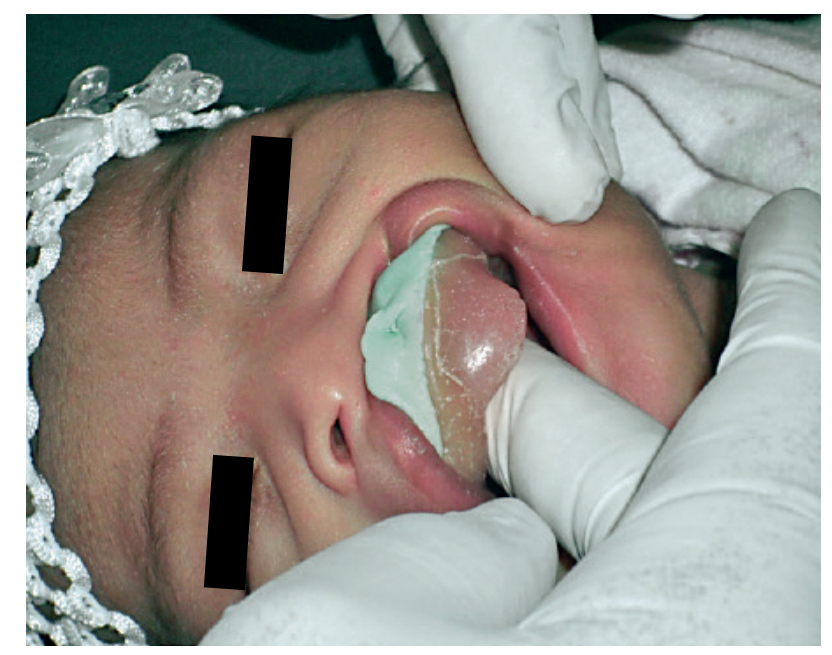

Figura 2. Registro del maxilar superior.

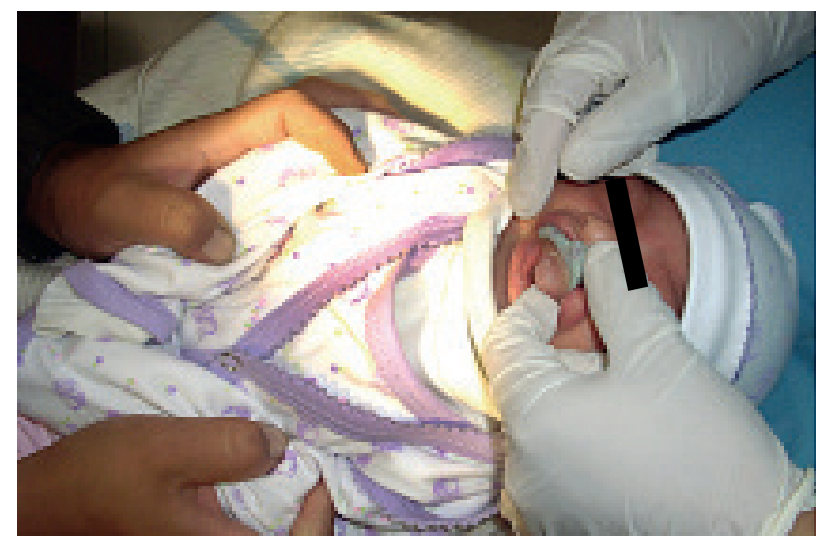

Figura 4. Registro del maxilar superior. El odontólogo se sienta frente a frente con la madre, juntando ambos las rodillas. La cabeza esta dirigida hacia el odontólogo y las piernas hacia la madre, quien además sostiene las manos.

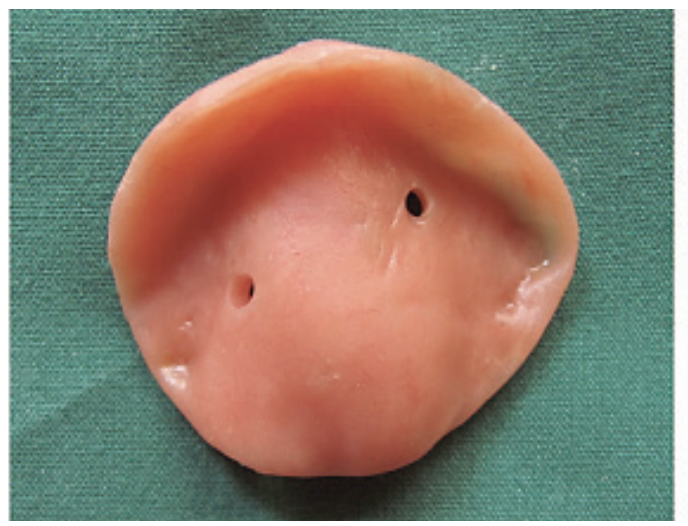

Figura 6. Cubeta para impresión.

- Putty (Zhermack), la cual se realizó mediante unas cubetas confeccionadas especialmente para neonatos (figura 5 y 6). Para vaciar la impresión se utilizó yeso tipo 4 (velmix).

En cada modelo obtenido de los casos de fisura labiopalatina unilateral, se dibujo con un lápiz 2 puntos, el punto " $A$ " en el proceso palatino del lado sano y el 


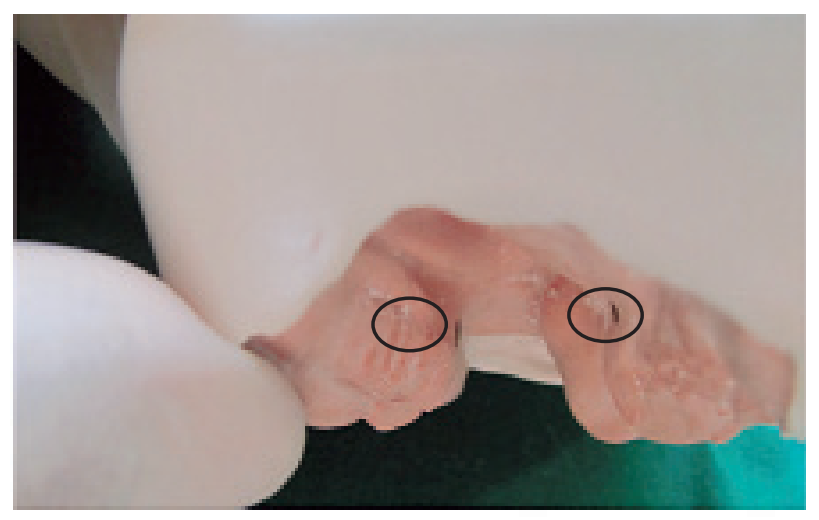

Figura 7. Ubicación del punto "A-B".

punto "B" en el proceso palatino del lado afectado (figura 7). El punto "A" visto frontalmente es el punto de unión más inferior y medial entre el paladar primario con el secundario a nivel de la hendidura y visto horizontalmente es el punto de unión mas anterior y medial entre el paladar primario con el secundario a nivel de la hendidura. El punto "B" visto frontalmente es el punto más superior a nivel de la hendidura y visto horizontalmente es el punto más anterior a nivel de la hendidura.

Se procedió a medir 2 veces con un calibrador digital (figura 8) la distancia existente entre el punto "A" y el punto "B", siendo el promedio obtenido de los 2 tiempos de medición representativo del nivel de fusión de los procesos palatinos.

Para determinar el grado de concordancia en la ubicación del punto "A-B", se realizó el "Juicio de Expertos" y la prueba de "Porcentaje de acuerdo entre los jueces". Para ello se solicitó la evaluación de 7 expertos conformados por cirujanos dentistas. Se confeccionó 7 réplicas de un modelo de un caso de fisura labiopalatina unilateral y a cada evaluador se le entregó un modelo. Cuando el evaluador finalizó la ubicación de los puntos en el modelo, se comparó con la ubicación que realizó el investigador

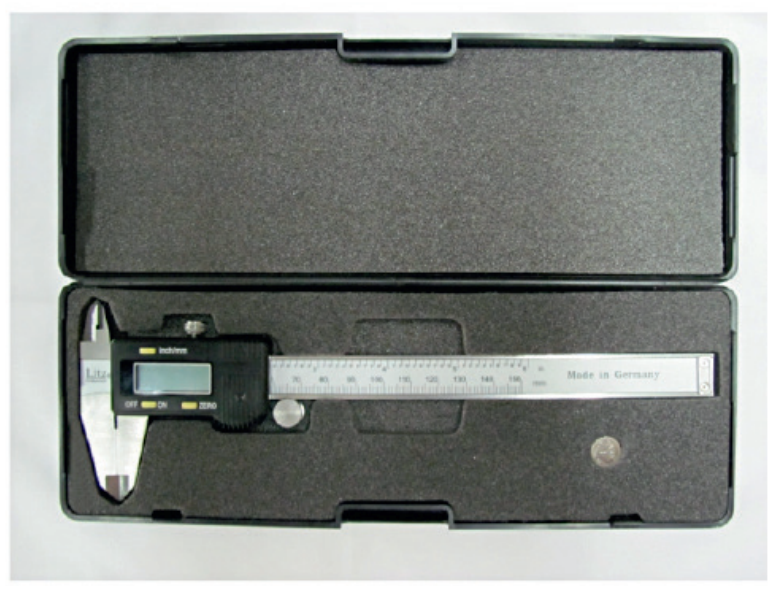

Figura 8. Calibrador digital. en el modelo original, se registró si el evaluador logró o no ubicar ambos puntos igual que el investigador y por último se registró la conformidad o no de la técnica. Se obtuvo un $100 \%$ de concordancia entre los jueces en la ubicación del punto "A" y $71,42 \%$ de concordancia entre los jueces en la ubicación del punto "B". Para evaluar la consistencia de las mediciones realizadas entre el punto "A - B" con el calibrador digital, se realizó la fiabilidad intra-examinador (1 investigador), para lo cual se midió cuantitativamente 2 veces la distancia entre el punto "AB" en los modelos obtenidos, al inicio, día 10 y día 20, en 4 casos y se aplicó la prueba de correlación intra-clase para determinar el grado de consistencia de las mediciones del antes y después; obteniendo como promedio de medida "1" al inicio , a los 10 días promedio "1" y a los 20 días promedio "1"; lo que indica una alta consistencia en las medidas obtenidas.

Así el día en que se realizó el registro de la ficha de recolección de datos se tomó la primera impresión y se obtuvo el primer modelo al cual se le realizó los procedimientos antes mencionados. El primer control se realizó en el día 10 y el segundo control en el día 20. En cada control se tomó una nueva impresión para obtener un nuevo modelo, a los cuales se les realizó el mismo procedimiento con la finalidad de comparar entre los tres modelos las medidas obtenidas en cada uno de ellos y así determinar el nivel de fusión de los procesos palatinos.

\section{Instrumento de recolección de datos}

Cada neonato seleccionado fue registrado en una ficha de recolección de datos, confeccionada especialmente para la presente investigación, en la cual se detalla el número de ficha, el número de historia clínica, la fecha en la cual se inicia el seguimiento, nombre del paciente, género (masculino o femenino), tipo de lactancia materna que recibiría el neonato (exclusiva o no exclusiva, registrándose en este ultimo caso la forma en que se alimentó al neonato), edad cronológica actual, indicadores del crecimiento al nacer tales como el peso (si nació con peso normal o bajo peso, se detalló los gr.); talla y perímetro cefálico (ambos en $\mathrm{cm}$.), indicadores del crecimiento al inicio del seguimiento, a los 10 días del inicio (día 10) y a los 20 días del inicio (día 20). Edad gestacional (nació a termino o pretérmino, se indicó las semanas), paridad de la madre (primípara ó multípara) y por último se registró la medida en milímetros del nivel de fusión de los procesos palatinos a nivel de la hendidura con un calibrador digital.

\section{Procesamiento y análisis de los datos}

El procesamiento de la información se realizó mediante un ordenador P4 y se utilizó el programa estadístico SPSS versión 18, el cual permitió presentar datos descriptivos y analíticos. Los resultados fueron presentados en tablas de $2 \times 3$, por la característica de la variable que compara con los controles (inicial, día 10 y día 20). Para el análisis estadístico, se realizó la prueba de normalidad K-S y Shapiro Wills, los cuales nos permitió comprobar la 
distribución normal de los datos. Para la comprobación de los datos de los controles que fueron 3 , se utilizó la prueba estadística de ANOVA y para comparar las medias en grupos de a dos, se utilizó la prueba estadística T-student para muestras relacionadas, ya que fueron grupos que se evaluaron consecutivamente. Para la contrastación de la hipótesis se consideró como parámetro de decisión un margen de error del $5 \%$, por lo tanto se trabajó con un nivel de confianza del $95 \%$.

\section{RESULTADOS}

En la tabla 1 correspondiente a las características al nacer según lactancia materna exclusiva y no exclusiva, de los neonatos con fisura labiopalatina unilateral, se obtuvo que; con respecto al género el 66,7 \% (2 casos) que nacieron de género masculino recibieron LMNE; mientras que el $60 \%$ (3 casos) que nacieron de género femenino recibieron LME. Con respecto al peso al nacer,

Tabla 1. Características al nacer según lactancia materna exclusiva y no exclusiva, de los neonatos con fisura labiopalatina unilateral.

\begin{tabular}{|c|c|c|c|c|c|}
\hline & & & LME & LMNE & TOTAL \\
\hline \multirow{6}{*}{ GÉNERO } & \multirow[t]{2}{*}{ Masculino } & $\mathbf{f}$ & 1 & 2 & 3 \\
\hline & & $\%$ & 33,3 & 66,7 & 100 \\
\hline & \multirow[t]{2}{*}{ Femenino } & $\mathbf{f}$ & 3 & 2 & 5 \\
\hline & & $\%$ & 60 & 40 & 100 \\
\hline & \multirow[t]{2}{*}{ TOTAL } & f & 4 & 4 & 8 \\
\hline & & $\%$ & 50 & 50 & 100 \\
\hline \multirow{4}{*}{ PESO AL N. } & \multirow[t]{2}{*}{ Normal } & f & 4 & 4 & 8 \\
\hline & & $\%$ & 50 & 50 & 100 \\
\hline & \multirow[t]{2}{*}{ TOTAL } & $\mathbf{f}$ & 4 & 4 & 8 \\
\hline & & $\%$ & 50 & 50 & 100 \\
\hline \multirow{6}{*}{ TALLA AL N. } & \multirow[t]{2}{*}{$45-49,5$} & f & 4 & 3 & 7 \\
\hline & & $\%$ & 57,1 & 42,9 & 100 \\
\hline & \multirow[t]{2}{*}{$49,6-55$} & f & 0 & 1 & 1 \\
\hline & & $\%$ & 0 & 100 & 100 \\
\hline & \multirow[t]{2}{*}{ TOTAL } & $\mathbf{f}$ & 4 & 4 & 8 \\
\hline & & $\%$ & 50 & 50 & 100 \\
\hline \multirow{6}{*}{ PC AL N. } & \multirow[t]{2}{*}{ Normocefália } & f & 4 & 3 & 7 \\
\hline & & $\%$ & 57,1 & 42,9 & 100 \\
\hline & \multirow[t]{2}{*}{ Megacefália } & $\mathbf{f}$ & 0 & 1 & 1 \\
\hline & & $\%$ & 0 & 100 & 100 \\
\hline & \multirow[t]{2}{*}{ TOTAL } & $\mathbf{f}$ & 4 & 4 & 8 \\
\hline & & $\%$ & 50 & 50 & 100 \\
\hline \multirow{6}{*}{ EDAD G. } & \multirow[t]{2}{*}{ Término } & $f$ & 3 & 3 & 6 \\
\hline & & $\%$ & 50 & 50 & 100 \\
\hline & \multirow[t]{2}{*}{ Pretérmino } & f & 1 & 1 & 2 \\
\hline & & $\%$ & 50 & 50 & 100 \\
\hline & \multirow[t]{2}{*}{ TOTAL } & $\mathbf{f}$ & 4 & 4 & 8 \\
\hline & & $\%$ & 50 & 50 & 100 \\
\hline \multirow{6}{*}{$\begin{array}{l}\text { PARIDAD DE } \\
\text { LA MADRE }\end{array}$} & \multirow[t]{2}{*}{ Primípara } & $f$ & 3 & 2 & 5 \\
\hline & & $\%$ & 60 & 40 & 100 \\
\hline & \multirow[t]{2}{*}{ Multípara } & $\mathbf{f}$ & 1 & 2 & 3 \\
\hline & & $\%$ & 33,3 & 66,7 & 100 \\
\hline & \multirow[t]{2}{*}{ TOTAL } & $\mathbf{f}$ & 4 & 4 & 8 \\
\hline & & $\%$ & 50 & 50 & 100 \\
\hline
\end{tabular}

Tabla 2. Prueba de normalidad de la variable nivel de fusión de los procesos palatinos.

\begin{tabular}{cccccccc}
\hline & \multicolumn{2}{c}{ Kolmogorov-Smirnov } & \multicolumn{3}{c}{ Shapiro-Wilk } \\
\hline & Estadístico & gl & Sig. & Estadístico & gl & Sig. \\
\hline Inicio & 0,186 & 8 & 0,200 & 0,960 & 8 & 0,813 \\
Día 10 & 0,188 & 8 & 0,200 & 0,946 & 8 & 0,671 \\
Día 20 & 0,185 & 8 & 0,200 & 0,946 & 8 & 0,669 \\
\hline
\end{tabular}

los 8 neonatos nacieron con peso normal. Con respecto a la talla al nacer, el $57,1 \%$ (4 casos) que presentaron una talla entre 45 a $49,5 \mathrm{~cm}$, recibieron LME; mientras que el $100 \%$ (1 caso) que presentó una talla entre 49.6 a 55 $\mathrm{cm}$, recibió LMNE. Con respecto al perímetro cefálico al nacer, el 57,1 \% (4 casos) que presentaron normocefália, recibieron LME; mientras que el $100 \%$ (1 caso) que presentó megacefálica, recibió LMNE. Con respecto a la edad gestacional, de los 6 casos que nacieron a término, el $50 \%$ recibieron LME y el otro $50 \%$ LMNE, de igual manera de los 2 casos que nacieron a pretérmino, el 50 $\%$ recibió LME y el otro $50 \%$ LMNE. Con respecto a la paridad de la madre, el $60 \%$ (3 casos) que recibieron LME nacieron de mujeres primíparas, mientras que el 66,7 $\%$ (2 casos) que recibieron LMNE nacieron de mujeres multíparas.

En la tabla 2 correspondiente a la prueba de normalidad de la variable nivel de fusión de los procesos palatinos, la distribución de las dimensiones en milímetros en los diferentes periodos de lactancia, inicio, día 10 y día 20, cumplen una distribución de normalidad.

En la tabla 3 correspondiente al nivel de fusión de los procesos palatinos según periodo de lactancia, en neonatos con fisura labiopalatina unilateral que recibieron lactancia materna exclusiva y no exclusiva, se observa que, al inicio del seguimiento el valor mínimo y el máximo del nivel de fusión estuvieron entre 2,38 mm a 14,37 mm respectivamente, encontrando que el promedio en los 8 casos fue de 9,05 mm. En el día 10 después de iniciado el seguimiento, los niveles de fusión variaron entre 1,98 mm a $14,29 \mathrm{~mm}$, con un promedio de $7,75 \mathrm{~mm}$. Finalmente en el día 20 después de iniciado el seguimiento, los niveles de fusión estuvieron entre $1,67 \mathrm{~mm}$ a $12,92 \mathrm{~mm}$ y el promedio fue de $6,69 \mathrm{~mm}$. Sin embargo dichas diferencias no son estadísticamente significativas.

Tabla 3. Nivel de fusión de los procesos palatinos según periodo de lactancia, en neonatos con fisura labiopalatina unilateral que recibieron lactancia materna exclusiva y no exclusiva.

\begin{tabular}{lcccccc}
\hline & N & Media & D. Estandar & Mínimo & Máximo & Sig. * \\
\hline Inicio & 8 & 9,0525 & 3,75443 & 2,38 & 14,37 & \\
Día 10 & 8 & 7,7538 & 3,97365 & 1,98 & 14,29 & 0,489 \\
Día 20 & 8 & 6,6913 & 3,93725 & 1,67 & 12,92 & \\
\hline *ANOVA. & & & & & \\
\end{tabular}


Tabla 4. Nivel de fusión de los procesos palatinos según periodo de lactancia, en neonatos con fisura labiopalatina unilateral que recibieron lactancia materna exclusiva.

\begin{tabular}{lrrrrr}
\hline & N & Media & D. Estandar & Anova & Sig \\
& & & & & \\
\hline Inicial & 4 & 10,6050 & 2,58942 & & \\
Día 10 & 4 & 9,1250 & 3,70479 & 0,503 & 0,621 \\
Día 20 & 4 & 8,1650 & 3,95306 & & \\
\hline
\end{tabular}

En la tabla 4 correspondiente al nivel de fusión de los procesos palatinos según periodo de lactancia, en neonatos con fisura labiopalatina unilateral que recibieron lactancia materna exclusiva; el nivel de fusión fue aumentando progresivamente: $10.60 \mathrm{~mm}, 9.12 \mathrm{~mm}$ y 8.16 $\mathrm{mm}$, sin embargo dichas diferencias de promedios no son estadísticamente significativas.

En la tabla 5 correspondiente al nivel de fusión de los procesos palatinos según periodo de lactancia, en neonatos con fisura labiopalatina unilateral que recibieron lactancia materna no exclusiva; el nivel de fusión fue aumentando progresivamente: $7,5 \mathrm{~mm}, 6,38 \mathrm{~mm}$ y 5,21 $\mathrm{mm}$; sin embargo dichas diferencias de promedios no son estadísticamente significativas.

En la tabla 6 correspondiente a los indicadores del crecimiento entre los diferentes periodos de lactancia, en neonatos con fisura labiopalatina unilateral que recibieron lactancia materna exclusiva, se tuvo que; con respecto al peso, al inicio del seguimiento el promedio fue de 2987 gr. Luego de 10 días de iniciado el seguimiento el promedio aumentó a $3020 \mathrm{gr}$; siendo la diferencia de promedios entre el inicio del seguimiento y el control del día 10, no significativa estadísticamente. Luego de 20 días de iniciado el seguimiento, el promedio aumentó a $3212 \mathrm{gr}$; siendo la diferencia de promedios entre el inicio del seguimiento y el control del día 20, no significativa estadísticamente. De igual manera, la diferencia de promedios entre el control del día 10 y del día 20, no es significativa estadísticamente. Con respecto a la talla, al inicio del seguimiento el promedio fue de $47,75 \mathrm{~cm}$. Luego de 10 días de iniciado el seguimiento el promedio aumentó a $49 \mathrm{~cm}$; siendo la diferencia de promedios entre el inicio del seguimiento y el control del día 10, significativa

Tabla 5. Nivel de fusión de los procesos palatinos según periodo de lactancia, en neonatos con fisura labiopalatina unilateral que recibieron lactancia materna no exclusiva.

\begin{tabular}{lccccc} 
& N & Media & D. Estandar & Anova & Sig. \\
& & & & & \\
\hline Inicio & 4 & 7,5000 & 4,44494 & & \\
Día 10 & 4 & 6,3825 & 4,25482 & 0,297 & 0,750 \\
Día 20 & 4 & 5,2175 & 3,84092 & & \\
\hline
\end{tabular}

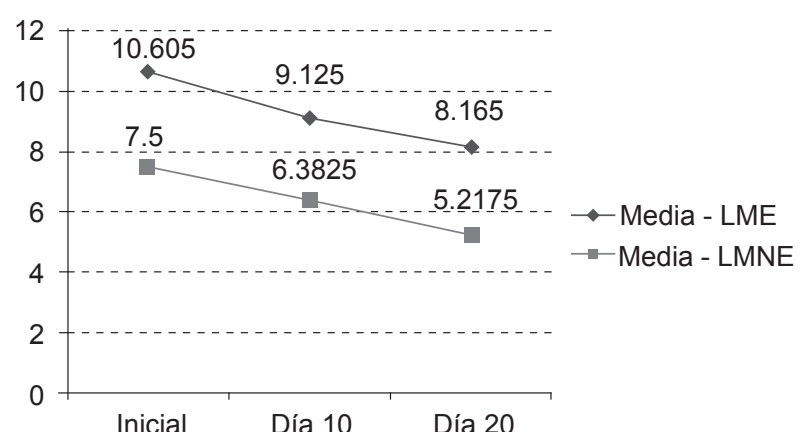

Figura 1. Nivel de fusión de los procesos palatinos según periodo de lactancia, en neonatos con fisura labiopalatina unilateral que recibieron lactancia materna exclusiva y no exclusiva, evaluando ambos grupos individualmente.

estadísticamente. Luego de 20 días de iniciado el seguimiento, el promedio aumentó a 49,125 cm; siendo la diferencia de promedios entre el inicio del seguimiento y el control del día 20, significativa estadísticamente. Por lo contrario, la diferencia de promedios entre el control del día 10 y del día 20 , no es significativa estadísticamente. Con respecto al perímetro cefálico, al inicio del seguimiento el promedio fue de $34 \mathrm{~cm}$. Luego de 10 días de iniciado el seguimiento el promedio aumentó a $34,5 \mathrm{~cm}$; siendo la diferencia de promedios entre el inicio del seguimiento y el control del día 10, no significativa estadísticamente. Luego de 20 días de iniciado el seguimiento, el promedio aumentó a $35,25 \mathrm{~cm}$; siendo la diferencia de promedios entre el inicio del seguimiento y el control del día 20, significativa estadísticamente. En cuanto a la diferencia de promedios entre el control del día 10 y del día 20 , no es significativa estadísticamente.

Tabla 6. Indicadores del crecimiento entre los diferentes periodos de lactancia, en neonatos con fisura labiopalatina unilateral que recibieron lactancia materna exclusiva.

\begin{tabular}{|c|c|c|c|c|c|c|}
\hline & & $N$ & Media & $\begin{array}{c}\text { D. } \\
\text { Estandar }\end{array}$ & $\begin{array}{c}\mathrm{T} \\
\text { student }\end{array}$ & Sig. \\
\hline \multirow{6}{*}{ Peso } & Inicio & 4 & 2987,5 & 197,379 & $-0,477$ & 0,666 \\
\hline & Día 10 & 4 & 3020 & 285,657 & & \\
\hline & Inicio & 4 & 2987,5 & 197,379 & $-2,435$ & 0,093 \\
\hline & Día 20 & 4 & 3212,5 & 347,311 & & \\
\hline & Día 10 & 4 & 3020 & 285,657 & $-1,433$ & 0,247 \\
\hline & Día 20 & 4 & 3212,5 & 347,311 & & \\
\hline \multirow{6}{*}{ Talla } & Inicio & 4 & 47,75 & 0,2887 & $-8,66$ & 0,003 \\
\hline & Día 10 & 4 & 49 & 0 & & \\
\hline & Inicio & 4 & 47,75 & 0,2887 & -11 & 0,002 \\
\hline & Día 20 & 4 & 49,125 & 0,25 & & \\
\hline & Día 10 & 4 & 49 & 0 & -1 & 0,391 \\
\hline & Día 20 & 4 & 49,125 & 0,25 & & \\
\hline \multirow{6}{*}{$\mathrm{Pc}$} & Inicio & 4 & 34 & 0,7071 & $-2,449$ & 0,092 \\
\hline & Día 10 & 4 & 34,5 & 0,5 & & \\
\hline & Inicio & 4 & 34 & 0,7071 & $-8,66$ & 0,003 \\
\hline & Día 20 & 4 & 35,25 & 0,9574 & & \\
\hline & Día 10 & 4 & 34,5 & 0,5 & -3 & 0,058 \\
\hline & Día 20 & 4 & 35,25 & 0,9574 & & \\
\hline
\end{tabular}


En la tabla 7 correspondiente a los indicadores del crecimiento entre los diferentes periodos de lactancia, en neonatos con fisura labiopalatina unilateral que recibieron lactancia materna no exclusiva, se tuvo que; con respecto al peso, al inicio del seguimiento el promedio fue de 3337 gr. Luego de 10 días de iniciado el seguimiento el promedio disminuyó a $3280 \mathrm{gr}$. Siendo la diferencia de promedios entre el inicio del seguimiento y el control del día 10, no significativa estadísticamente. Luego de 20 días de iniciado el seguimiento el promedio disminuyó a 3275 gr; siendo la diferencia de promedios entre el inicio del seguimiento y el control del día 20, no significativa estadísticamente. De igual manera la diferencia de promedios entre el control del día 10 y del día 20, no es significativa estadísticamente. Con respecto a la talla, al inicio del seguimiento el promedio fue de $49,5 \mathrm{~cm}$. Luego de 10 días de iniciado el seguimiento el promedio aumentó a $50,5 \mathrm{~cm}$; siendo la diferencia de promedios entre el inicio del seguimiento y el control del día 10, significativa estadísticamente. Luego de 20 días de iniciado el seguimiento el promedio aumentó a $51 \mathrm{~cm}$; siendo la diferencia de promedios entre el inicio del seguimiento y el control del día 20, significativa estadísticamente. Por lo contrario la diferencia de promedios entre el control del día 10 y del día 20 , no es significativa estadísticamente. Con respecto al perímetro cefálico, al inicio del seguimiento el promedio fue de $34,87 \mathrm{~cm}$. Luego de 10 días de iniciado el seguimiento el promedio aumentó a $35,25 \mathrm{~cm}$; siendo la diferencia de promedios entre el inicio del seguimiento y el control del día 10, no significativa estadísticamente. Luego de 20 días de iniciado el seguimiento el promedio aumentó a $35,62 \mathrm{~cm}$; siendo la diferencia de promedios entre el inicio del seguimiento y el control del día 20, no significativa estadísticamente. En cuanto a la diferencia de promedios entre el control del día 10 y del día 20 , no es significativa estadísticamente.

Tabla 7. Indicadores del crecimiento entre los diferentes periodos de lactancia, en neonatos con fisura labiopalatina unilateral que recibieron lactancia materna no exclusiva.

\begin{tabular}{lllrrrr}
\hline & & N & Media & D. Estandar & $\begin{array}{c}\text { T } \\
\text { student }\end{array}$ & Sig. \\
\hline \multirow{4}{*}{ Peso } & Inicio & 4 & 3337,5 & 469,494 & 0,546 & 0,623 \\
& Día 10 & 4 & 3280 & 459,275 & & \\
& Inicio & 4 & 3337,5 & 469,494 & 0,325 & 0,766 \\
& Día 20 & 4 & 3275 & 359,398 & & \\
& Día 10 & 4 & 3280 & 459,275 & 0,042 & 0,969 \\
& Día 20 & 4 & 3275 & 359,398 & & \\
& Inicio & 4 & 49,5 & 0,6124 & $-4,899$ & 0,016 \\
& Día 10 & 4 & 50,5 & 0,6455 & & \\
Talla & Inicio & 4 & 49,5 & 0,6124 & -23 & 0,000 \\
& Día 20 & 4 & 51 & 0,6124 & & \\
& Día 10 & 4 & 50,5 & 0,6455 & $-2,449$ & 0,092 \\
& Día 20 & 4 & 51 & 0,6124 & & \\
& Inicio & 4 & 34,875 & 0,9656 & $-1,567$ & 0,215 \\
& Día 10 & 4 & 35,25 & 1,031 & & \\
Pc Inicio & 4 & 34,875 & 0,9656 & -3 & 0,058 \\
& Día 20 & 4 & 35,625 & 0,8004 & & \\
& Día 10 & 4 & 35,25 & 1,031 & -1.567 & 0,215 \\
& Día 20 & 4 & 35,625 & 0,8004 & & \\
\hline
\end{tabular}

\section{DISCUSIÓN}

La fisura labiopalatina unilateral se presentó en un 62,5 $\%$ en el género femenino, siendo este género el que tiene mayor tendencia a la fisura labiopalatina unilateral; lo cual genera discordancia con los resultados obtenidos por Rojas $\mathrm{GE}^{10}$ y Bascones $\mathrm{A}^{2}$ en su libro Medicina Bucal, quienes mencionan que se presenta con mayor frecuencia en el género masculino. Al nacer el $100 \%$ de los casos con peso normal, no se puede determinar si existe o no relación entre el peso al nacer con la tendencia a algún tipo de lactancia materna; al presentarse los casos con peso normal de manera equitativa entre la LME y LMNE, lo que se puede interpretar es que cuando los neonatos con fisura labiopalatina unilateral nacen con peso normal no influye en que no pueda recibir LME; aunque no se han encontrado estudios que muestren similitud o contrariedad con los cuales se pueda discutir. Con respecto a la talla, en un $87,5 \%$ los neonatos nacieron con una talla entre $45-49,5 \mathrm{~cm}$ y de este grupo el $57,1 \%$ tuvieron tendencia a LME; mientras que el $22,5 \%$ del total de casos, nació con una talla superior a los $49,5 \mathrm{~cm}$ y presentó tendencia a la LMNE; lo que demuestra que el neonato no tiene que estar necesariamente bien desarrollado para que pueda recibir LME y en realidad la talla al nacer no influye en que no pueda recibir LME; aunque no se han encontrado estudios que muestren similitud o contrariedad con los cuales se pueda discutir. En un $87,5 \%$ los neonatos nacieron con normocefália y de este grupo el $57,1 \%$ tuvo tendencia a LME; mientras que el $22,5 \%$ del total de casos, nació con megacefália y presentó tendencia a LMNE; por tanto se podría interpretar que hay una mayor tendencia a la LME en los neonatos con fisura labiopalatina unilateral que nacen con normocefália, pero en realidad el perímetro cefálico no influye en que no pueda recibir LME; aunque no se han encontrado estudios que muestren similitud o contrariedad con los cuales se pueda discutir. Así pues, al determinar que los indicadores del crecimiento al nacer, no influyen en que el neonato con fisura labiopalatina unilateral no pueda recibir LME y sabiendo que la antropometría del recién nacido es indicador importante de la salud fetal, neonatal y posneonatal tal como lo indican Mc Cormick y la National Academy of Sciences of Washington DC; la presente investigación comparte lo declarado por la OMS y la UNICEF ${ }^{8}$, que es promover la LME ya que es la forma ideal y natural de alimentar al lactante al constituir la base biológica para su desarrollo. En un $75 \%$ los neonatos nacieron a término, pero tanto los neonatos que nacieron a término y a pretérmino tuvieron tendencia a recibir LME o LMNE indistintamente, por tanto el hecho de que el neonato haya nacido a pretérmino no influye en que no pueda recibir LME. Los neonatos nacieron en un 62,5 \% de mujeres primíparas, presentando tendencia en un $60 \%$ a LME (figuras 9 y 10); por el contrario los neonatos que nacieron de mujeres multíparas tuvieron en un 66,7 \% tendencia a LMNE; con lo cual se puede interpretar que las mujeres primíparas tienen mayor afinidad, cuidado e interés por su hijo(a); aunque no se han encontrado estudios que muestren similitud o contrariedad con los cuales se pueda discutir. 
En cuanto al nivel de fusión de los procesos palatinos, al analizar los niveles de forma global juntando los casos con LME y LMNE, se puede apreciar que desde el inicio del seguimiento hasta el día 10 se logró aumentar $2 \mathrm{~mm}$ el nivel de fusión, mientras que desde el inicio hasta el día 20 se logró un aumento total de $2,36 \mathrm{~mm}$; con esto se demuestra que conforme el niño va creciendo se logra aumentar el nivel de fusión de los procesos palatinos; generando contrariedad significativa con respecto a lo que menciona Navarro $\mathrm{C}^{3}$ en su libro Tratado de cirugía oral y maxilofacial; ya que el menciona que el hemimaxilar del lado sano presenta un desarrollo armónico, mientras que el hemimaxilar del lado afectado, se encuentra hipoplásico y retrusivo; al no presentar una buena inserción muscular y acción protrusiva de la lengua; obteniendo como resultado la amplitud de la hendidura durante el crecimiento. Al comparar el nivel de fusión de los procesos palatinas solo en los neonatos que recibieron LME, se puede apreciar que desde el inicio del seguimiento al día 10, se logró aumentar 1,48 mm; mientras que al comparar desde el día 10 al día 20 , se logró aumentar $0,96 \mathrm{~mm}$, obteniendo en el día 20 un aumento total de $2,44 \mathrm{~mm}$; sin embargo dichas diferencias no son estadísticamente significativas. Al comparar el nivel de fusión de los procesos palatinos en los neonatos que recibieron LMNE, se puede apreciar que desde el inicio del seguimiento al día 10 se logró aumentar $1,11 \mathrm{~mm}$, mientras que al comparar desde el día 10 al día 20, se logró aumentar 1,16 mm, obteniendo en el día 20 un aumento total de 2,28 mm; sin embargo dichas diferencias no son estadísticamente significativas. No obstante, si comparamos clínicamente el aumento total del nivel de fusión a los 20 días, entre los neonatos que recibieron LME y LMNE, se aprecia que se logra un mayor aumento en aquellos que recibieron LME; confirmando lo que mencionan diversos autores como Vera $\mathrm{H}$ y Col${ }^{6}$, Pilonieta $\mathrm{G}$ y $\mathrm{Col}{ }^{11}$, quienes mencionan que la LME estimula el desarrollo del sistema estomatognático y el complejo craneofacial. Así también, relacionándose con el estudio de Blanco y Cols $^{8}$, quienes encontraron la tendencia de la LMNE a generar paladar profundo producto de un inadecuado estimulo para el desarrollo del maxilar superior.

El promedio del peso en los neonatos que recibieron LME, desde el inicio del seguimiento hasta el día 20, aumentó $225 \mathrm{gr}$, sin embargo esta diferencia no es estadísticamente significativa. Por lo contrario, el promedio del peso en los neonatos que recibieron LMNE, desde el inicio del seguimiento hasta el día 20, disminuyó $62 \mathrm{gr}$, sin embargo esta diferencia no es estadísticamente significativa. No obstante, se puede apreciar notoriamente que los neonatos que reciben LME se nutren mejor y por ende aumentan de peso; mientras que los neonatos que reciben LMNE al no tener una buena nutrición bajan de peso. Además, si tenemos en cuenta lo que Gonzales ${ }^{12}$ menciona en su investigación, de que generalmente los recién nacidos pierden hasta un $10 \%$ de su peso los primero 10 a 15 días, siendo esos valores considerados

algo normal, podemos darnos cuenta en base a la evidencia que la presente investigación muestra, que los neonatos alimentados con LME pertenecen al poco grupo de recién nacidos que en lugar de bajar, aumentan de peso. Cuando se compara el promedio de la talla desde el inicio del seguimiento hasta el día 20 , se puede apreciar que se genera un aumento estadísticamente significativo indistintamente del tipo de lactancia materna que reciba. Al comparar el promedio del perímetro cefálico desde el inicio del seguimiento hasta el día 20 , en los neonatos que recibieron $\mathrm{LME}$, se obtiene que hubo un aumento de 1,25 $\mathrm{cm}$, mostrando diferencia estadísticamente significativa; por lo contrario en los neonato que recibieron LMNE el aumento fue de $0,75 \mathrm{~cm}$, lo cual no muestra diferencia estadísticamente significativa. Lo que demuestra que la lactancia materna exclusiva estimula un mayor aumento del perímetro cefálico y por ende un mayor crecimiento del complejo cráneo-maxilo-facial; relacionándose con los hallazgos encontrados por Berrocal ${ }^{13}$, quien menciona que los niños con amamantamiento fisiológico (LME) tienen tendencia a ser mesocéfalos y presentar facie mesofacial.

La evidencia científica encontrada en el presente estudio demuestra que la LME es la que estimula una mayor fusión de los procesos palatinos, en comparación a la LMNE; aunque con una diferencia estadísticamente no significativa.

\section{CONCLUSIONES}

a. La LME es la que estimula una mayor fusión de los procesos palatinos en comparación a la LMNE; aunque con una diferencia estadísticamente no significativa.

b. La fisura labiopalatina unilateral se presenta con mayor frecuencia en el género femenino.

c. Las mujeres primíparas son las que tienen mayor tendencia a alimentar a sus hijos con LME.

d. La LME genera ganancia de peso mientras que la LMNE genera perdida de peso.

e. La LME genera un mayor estimulo estadísticamente significativo para el aumento del perímetro cefálico y por ende para el crecimiento del complejo craneomaxilo-facial.

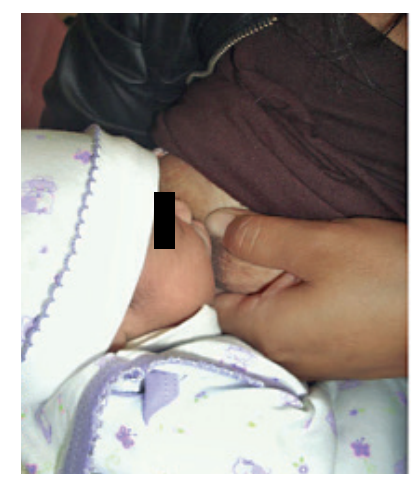

Figura 9. Neonato con FLP-U Figura 10. Neonato con FLP-B recibiendo LME.

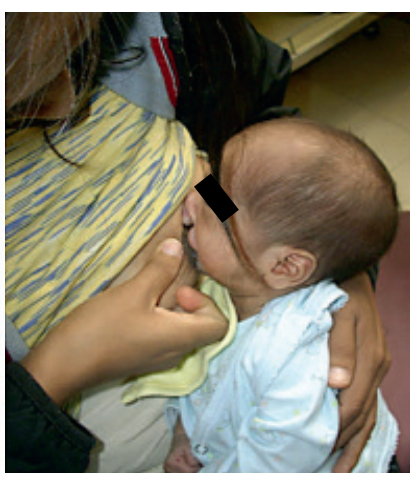

recibiendo LME. 


\section{REFERENCIAS BIBLIOGRÁFICAS}

1. Philip J, Eversole LR, Wysocki GP. Patologia oral y maxilofacial contemporánea. 2a. ed. Madrid: Ed. Harcourt. Cap. 1: 27-9.

2. Bascones A. Medicina bucal. $3^{a}$. Ed. Barcelona: Ed. Ariel. Capítulo 13-14: 280- 81; 306- 07.

3. Navarro C. Tratado de cirugía oral y maxilofacial. Madrid: Ed. Aran. 2004. Capitulo 48: 815 - 836. Cap. 51:871-8.

4. Lagman. Embriología Médica. 10a edición. Buenos Aires: Ed. Panamericana. 2007.

5. Berry K. Abordaje de Pacientes con Defectos Faciales (Congénitos, Tumorales y Traumáticos) en Jornadas Quirúrgicas de Cirugía Plástica y Maxilofacial realizadas en el H.E.O.D.R.A. del 2007 al 2009. Tesis de la Facultad de Ciencias Médicas de la Universidad Nacional Autónoma de Nicaragua. 2009.

6. Vera HD, Hess G. Recomendaciones actuales sobre lactancia materna. Revista Mexicana de pediatría; 2007; 74 (4): 176 81.

7. López P, Arévalo $\mathrm{B}$, Carrero R, Martínez J, Lorente $\mathrm{T}$, Rodríguez $A$. La lactancia natural o materna: conceptos básicos y trascendencia actual. 2000. Disponible en: www. medieneral. org/abril2000/360-369.pdf.

8. Merino E. Lactancia materna y su relación con las anomalías dento -faciales. Revisión de la literatura. Acta odontológica venezolana 2003; 41.

9. Garcez LW, Giuguani ERJ. Population-Based Study on the Practice of Breastfeeding in Children Born With Cleft Lip and Palate. Clell Palate-Craniofacial Journal; 2005; 42 (6): 687 93.

10. Rojas GE. Estudio de la deglución y de foniatría en niños con labio leporino y fisura palatina después de la intervención quirúrgica en la provincia de Moyobamba - departamento de San Martín. Tesis de la F. O. de la USMP. 2006.

11. Pilonieta G, Torres EA. Implicaciones de la lactancia materna en odontopediatría. Medunab; 2003; 6 (17): 89 - 2.

12. Gonzales A, Pupo L. Estado de la calidad de los cuidados nutricionales en la unidad de terapia intensiva neonatal. Rev Cubana Aliment Nutr 2008; 18 (1): 53 - 71.

13. Berrocal K. Estudio comparativo de la influencia del amamantamiento fisiológico y de biberón en la oclusión de niños con 3 a 5 años. Tesis de la Facultad de Obstetricia de la Universidad San Martín de Porres. 1995.

Correspondencia: Yoshio Dakusaku López

Correo electrónico: Yoshio_dakusaku@hotmail.com 